\title{
Obstructions for constructing equivariant fibrations
}

\author{
ASLI GÜÇLÜKAN İLHAN
}

Let $G$ be a finite group and $\mathcal{H}$ be a family of subgroups of $G$ which is closed under conjugation and taking subgroups. Let $B$ be a $G-\mathrm{CW}-$ complex whose isotropy subgroups are in $\mathcal{H}$ and let $\mathcal{F}=\left\{F_{H}\right\}_{H \in \mathcal{H}}$ be a compatible family of $H$-spaces. A $G$-fibration over $B$ with the fiber type $\mathcal{F}=\left\{F_{H}\right\}_{H \in \mathcal{H}}$ is a $G$-equivariant fibration $p: E \rightarrow B$ where $p^{-1}(b)$ is $G_{b}$-homotopy equivalent to $F_{G_{b}}$ for each $b \in B$. In this paper, we develop an obstruction theory for constructing $G$-fibrations with the fiber type $\mathcal{F}$ over a given $G-\mathrm{CW}$-complex $B$. Constructing $G$-fibrations with a prescribed fiber type $\mathcal{F}$ is an important step in the construction of free $G$-actions on finite $\mathrm{CW}$-complexes which are homotopy equivalent to a product of spheres.

57S25; 55R91

\section{Introduction}

In 1925, Hopf stated a problem which was later called the topological spherical space form problem: Classify all finite groups which can act freely on a sphere $\mathbb{S}^{n}, n>1$. One variant of this problem was solved by Swan [14]. He proved that a finite group acts freely on a finite complex homotopy equivalent to a sphere if and only if it has periodic cohomology. By using Swan's construction and surgery theory, the topological spherical space form problem has been completely solved by Madsen, Thomas and Wall [9]. It turns out that a finite group $G$ acts freely on a sphere if and only if $G$ has periodic cohomology and any element of order 2 in $G$ is central (see [9, Theorem 0.5]).

One of the generalizations of this problem is to classify all finite groups which can act freely on a finite $\mathrm{CW}$-complex homotopy equivalent to a product of $k$-spheres $\mathbb{S}^{n_{1}} \times \cdots \times \mathbb{S}^{n_{k}}$ for some $n_{1}, \ldots, n_{k}$. Recently, Adem and Smith [1] gave a homotopy theoretic characterization of cohomological periodicity and as a corollary they obtained a tool to construct free group actions on $\mathrm{CW}$-complexes homotopy equivalent to a product of spheres. More precisely, they have shown that a connected CW-complex $X$ has periodic cohomology if and only if there is a spherical fibration over $X$ with a total space $E$ that has a homotopy type of a finite dimensional CW-complex. As a consequence they proved that if $G$ is a finite group and $X$ is a finite dimensional $G-\mathrm{CW}$-complex whose isotropy subgroups all have periodic cohomology, then there 
is a finite dimensional CW-complex $Y$ with a free $G$-action such that $Y \simeq \mathbb{S}^{n} \times X$. As remarked in [1], the second result can also be obtained using the techniques given by Connolly and Prassidis in [3]. More recently, Klaus [6] proved that every $p$-group of rank 3 acts freely on a finite $\mathrm{CW}$-complex homotopy equivalent to a product of three spheres using similar techniques.

The method used by Connolly and Prassidis [3] is to construct a spherical fibration inductively over the skeleta by dealing with cells in each dimension separately. This is a standard strategy in obstruction theory. Note that if there is an orientable $G-$ spherical fibration over the $n$-skeleton of a CW-complex, then its restriction to the boundary of each $(n+1)$-cell $\sigma$ will be an orientable $G_{\sigma}$-fibration with the fiber $F_{G_{\sigma}}$, where $G_{\sigma}$ is the isotropy subgroup of $\sigma$. Associated to this $G_{\sigma}$-fibration over $\partial \sigma$, there is a classifying map from $\partial \sigma$ to the space $B$ Aut $_{G_{\sigma}} F_{G_{\sigma}}$ where Aut $_{G_{\sigma}} F_{G_{\sigma}}$ is the topological monoid of self $G_{\sigma}$-homotopy equivalences of $F_{G_{\sigma}}$. Combining the attaching map of $\sigma$ with the classifying map gives us an element in the $n$-th homotopy group of $B$ Aut $_{G_{\sigma}} F_{G_{\sigma}}$. Therefore we obtain a cellular cochain which assigns a homotopy class in $\pi_{n}\left(B\right.$ Aut $\left._{G_{\sigma}} F_{G_{\sigma}}\right)$ to each $(n+1)$-cell. This cochain vanishes if and only if the $G$-fibration over the $n$-skeleton extends to a $G$-fibration over the $(n+1)$-skeleton. In the situation Connolly and Prassidis consider, this cochain can be killed by taking fiber joins. Using this idea, Ünlü [17] gives a concrete cell-by-cell construction of $G$-spherical fibrations in his thesis.

In obstruction theory, one often has obstructions as cohomology classes which tells when a construction can be performed on the next skeleton after some modifications. In other words, the cohomological obstruction class vanishes if and only if the restriction of the construction to the $(n-1)$-skeleton extends over the $(n+1)$-skeleton. Having a cohomological obstruction is better than having a cochain class as an obstruction since a cohomology class is more likely to be zero. Note that if $p: E \rightarrow B$ is a $G$-fibration and $b \in B^{H}$ then the fiber $p^{-1}(b)$ is an $H$-space. When $B^{H}$ is connected for $H \leq G$, there is an $H$-space $F_{H}$ such that for every $b \in B^{H}$, the fiber $p^{-1}(b)$ is $H$-homotopy equivalent to $F_{H}$. Moreover, if $B^{H}$ is connected for every $H \leq G$, the family of $H$-spaces $F_{H}$ forms a compatible family (see Definition 2.5). In this case, the $G$-fibration $p: E \rightarrow B$ is said to have the fiber type $\left\{F_{H}\right\}$. In this paper, we notice that the cohomological obstructions for constructing $G$-fibrations with a given fiber type live in Bredon cohomology of $B$ with coefficients in $\underline{\pi}_{n, \mathcal{F}}$ (see Section 4 for the definition) and we prove the following theorem.

Theorem 1.1 Let $G$ be a finite group and $\mathcal{H}$ be a family of subgroups of $G$ which is closed under conjugation and taking subgroups. Let $B$ be a $G-C W$-complex whose isotropy subgroups are in $\mathcal{H}$ such that $B^{H}$ is simply connected for every 
$H \in \operatorname{Iso}(B)$. Let $\mathcal{F}=\left\{F_{H}\right\}_{H \in \mathcal{H}}$ be a compatible family of finite $H-C W$-complexes and $p: E_{n} \rightarrow B^{n}$ be a $G$-fibration over the $n$-skeleton of $B$ with the fiber type $\left\{F_{H}\right\}_{H \in \mathcal{H}}$ where $n \geq 2$.

(1) There is a cocycle $\alpha_{p} \in C_{\mathcal{H}}^{n+1}\left(B ; \underline{\pi}_{n, \mathcal{F}}\right)$ which vanishes if and only if $p$ extends to a $G$-fibration over $B^{n+1}$ with a total space $G$-homotopy equivalent to a $G-C W$-complex.

(2) The cohomology class $\left[\alpha_{p}\right] \in H_{G, \mathcal{H}}^{n+1}\left(B ; \underline{\pi}_{n, \mathcal{F}}\right)$ vanishes if and only if the $G-$ fibration $\left.p\right|_{B^{n-1}}: p^{-1}\left(B^{n-1}\right) \rightarrow B^{n-1}$ extends to a $G$-fibration over $B^{n+1}$ with a total space $G$-homotopy equivalent to a $G-C W$-complex.

Moreover if $B$ is a finite $G-C W$-complex then the total space of the obtained fibration has the $G$-homotopy type of a finite $G-C W$-complex whenever $E_{n}$ has the $G-$ homotopy type of a finite $G-C W-$ complex.

To prove this theorem we first define an obstruction cochain in the chain complex of Bredon cohomology and show that it is a cocycle. We call this cocycle an obstruction cocycle. Then we show that the difference of obstruction cocycles of any two extensions of the $G$-fibration $\left.p\right|_{B^{n-1}}$ is the coboundary of a cochain called the difference cochain. If there is an extension of $\left.p\right|_{B^{n-1}}$ to a $G$-fibration over $B^{n+1}$, then the obstruction cocycle of the restriction of this extension to $B^{n}$ vanishes. This means that the obstruction cocycle of $p$ is a coboundary and represents a cohomology class which vanishes. This proves the "if" direction of the above theorem.

For the "only if" direction it suffices to show that for every cochain $d$ there is a $G-$ fibration $q$ over $B^{n}$ with $\left.q\right|_{B^{n-1}}=\left.p\right|_{B^{n-1}}$ such that $d$ is the difference cochain of the extensions $p$ and $q$ of $\left.p\right|_{B^{n-1}}$. Here the most technical part is the construction of a $G$-fibration $q$ with these properties. That is because it is not clear how to glue $G$-fibration $\left.p\right|_{B^{n-1}}$ to $G$-fibrations over the $n$-cells corresponding to the cochain $d$. For quasifibrations it suffices to take the adjunction of the total spaces to glue two quasifibrations over different base. However, in order to obtain a fibration one needs to put some $G$-tubes between total spaces of these $G$-fibrations to create enough space to deal with $G$-homotopies. We use a generalization of a result due to Tulley [16] to produce a $G$-fibration $q$ with the required properties.

The paper is organized as follows: Section 2 contains definitions and preliminary results on equivariant fibrations. In Section 3, we give a method to glue $G$-fibrations over different base spaces by generalizing a construction due to Tulley [16]. Finally, we prove Theorem 1.1 in Section 4. 


\section{Equivariant fibrations}

In this section, we give the basic definitions of the equivariant fibration theory. We refer the reader to Lück [8] and Waner [19] for more details.

Definition 2.1 Let $G$ be a finite group. A $G$-map $p: E \rightarrow B$ is called a $G$-fibration if it has the $G$-homotopy lifting property with respect to every $G$-space $X$, that is, given a commutative diagram of $G$-maps

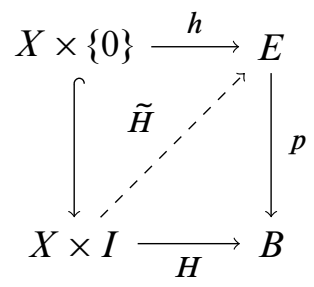

there exists a $G$-map $\widetilde{H}: X \times I \rightarrow E$ such that $p \widetilde{H}=H$ and $\left.\widetilde{H}\right|_{X \times\{0\}}=h$.

Equivalently, a $G$-map $p: E \rightarrow B$ is a $G$-fibration if there is a $G$-map

$$
\lambda: \Omega_{p}=\left\{(e, \omega) \in E \times B^{I} \mid p(e)=\omega(0)\right\} \rightarrow E^{I}
$$

such that $\lambda(e, \omega)(0)=e$ and $p \lambda(e, \omega)=\omega$. The $G$-map $\lambda$ is called a $G$-lifting function. In the nonequivariant theory, Dold [4] proved that being a fibration is a local property. The same proof applies to the equivariant case.

Definition 2.2 A covering $\mathcal{U}$ of $G$-invariant open sets of $B$ is called numerable $G$-covering if $\mathcal{U}$ is locally finite and there is a $G$-map $f_{U}: B \rightarrow I$ such that $U=$ $f_{U}^{-1}(0,1]$ for every $U \in \mathcal{U}$.

Theorem 2.3 A G-map $p: E \rightarrow B$ is a $G$-fibration if there is a numerable $G-$ covering $\mathcal{U}$ of $B$ such that $\left.p\right|_{U}: p^{-1}(U) \rightarrow U$ is a $G$-fibration for each $U \in \mathcal{U}$.

The notion of an equivalence between $G$-fibrations is defined naturally as follows: Let $p_{i}: E_{i} \rightarrow B$ be a $G$-fibration for $i=1,2$. A fiber preserving $G$-map $f: E_{1} \rightarrow E_{2}$ is called a $G$-fiber homotopy equivalence if there is a fiber preserving $G$-map $g: E_{2} \rightarrow E_{1}$ such that the compositions $f g$ and $g f$ are $G$-homotopy equivalent to identity maps through $G$-homotopies which are fiber preserving at each time $t \in I$. In this case, we write $p_{1} \simeq_{G} p_{2}$. As in the nonequivariant case, a fiber preserving $G$-homotopy equivalence between $G$-fibrations is a $G$-fiber homotopy equivalence (see May [10, page 50] for the proof of the nonequivariant case). 
In [12], Stasheff proved a classification theorem for nonequivariant fibrations up to fiber homotopy equivalences. When a $G$-fibration is over a path-connected space with trivial $G$-action, the fiber at each point in the base has a natural $G$-space structure and all fibers are $G$-homotopy equivalent with respect to this structure. In this case, the theory of $G$-fibrations is essentially the same as the nonequivariant one and we have the following classification theorem.

Theorem 2.4 Let $\operatorname{Aut}_{G}\left(F_{G}\right)$ be the monoid of $G$-equivariant self homotopy equivalences of a finite $G-C W$-complex $F_{G}$. If $B$ is a $C W$-complex with trivial $G$-action then there is a one-to-one correspondence between the set of $G$-fiber homotopy equivalence classes of $G$-fibrations $p: E \rightarrow B$ with fibers having the $G$-homotopy type of $F_{G}$ and the set of homotopy classes of maps $B \rightarrow B \operatorname{Aut}_{G}\left(F_{G}\right)$. The equivalence is obtained by taking the pullback fibration from the universal fibration over $B \operatorname{Aut}_{G}\left(F_{G}\right)$.

This classification theorem can be proved by using the same techniques and ideas from Stasheff [12]. Also, Waner constructs a classifying space for a more general set of equivariant fibrations in [18] and the above theorem can be obtained as a special case of his result.

The monoid $\operatorname{Aut}_{G}\left(F_{G}\right)$ is not connected in general. However, its connected compo-

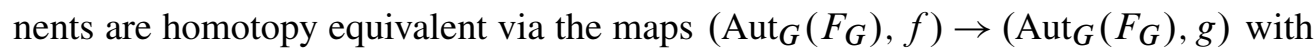
$\phi \rightarrow g \phi f^{-1}$ where $f^{-1}$ is the homotopy inverse of $f$. Furthermore, when the map $\pi_{1}(B) \rightarrow\left[F_{G}, F_{G}\right]_{G}$ is trivial, $B \operatorname{Aut}_{G}^{I}\left(F_{G}\right)$ classifies $G$-fibrations $p: E \rightarrow B$ with trivial $G$-actions on the base where $\operatorname{Aut}_{G}^{I}\left(F_{G}\right)$ is the connected component of identity in $\operatorname{Aut}_{G}\left(F_{G}\right)$ (see Adem and Smith [1] for nonequivariant case).

For $G$-fibrations whose $G$-action on the base is not trivial, we need to consider the collection of equivariant spaces. Note that if $p$ is a $G$-fibration over $B$ and $b \in B^{K}$, then the space $p^{-1}(b)$ is closed under $K$-action and hence a $K$-space. Moreover, when $B^{K}$ is connected, the spaces $p^{-1}(b)$ and $p^{-1}\left(b^{\prime}\right)$ are $K$-homotopy equivalent for every $b, b^{\prime} \in B^{K}$. On the other hand, when $H^{a} \leq K$, we have $h(a b)=a\left(a^{-1} h a\right) b=a b$ for every $h \in H$ and $b \in B^{K}$, hence $a b \in B^{H}$. Clearly, the $H$-space $p^{-1}(b)$, where the $H$-action on $p^{-1}(b)$ is given by conjugation and the $H$-space $p^{-1}(a b)$ are $H$-homeomorphic. Therefore, when $B^{H}$ is connected for every isotropy subgroup $H$ of $B$, the spaces $p^{-1}(b)$ and $p^{-1}(a b)$ are $H$-homotopy equivalent for every isotropy subgroups $H, K$ with $H^{a} \leq K$.

Definition 2.5 Let $\mathcal{H}$ be a family of subgroups of $G$ which is closed under conjugation and taking subgroups. A family $\mathcal{F}=\left\{F_{H}\right\}_{H \in \mathcal{H}}$ of $H$-spaces is said to be a compatible family if $F_{H}$ is $H$-homotopy equivalent to $F_{K}$ for every $H, K \in \mathcal{H}$ with $H^{a} \leq K$ for some $a \in G$ where the $H$-action on $F_{K}$ is given by $h \cdot y=a^{-1} h a y$. 
Let $\mathcal{F}=\left\{F_{H}\right\}_{H \in \mathcal{H}}$ be a compatible family where $\mathcal{H}$ contains the isotropy subgroups of $B$. We say $p: E \rightarrow B$ is a $G$-fibration with the fiber type $\mathcal{F}=\left\{F_{H}\right\}_{H \in \mathcal{H}}$ if $F_{H} \simeq_{H} p^{-1}(b)$ for every $b \in B^{H}$ and for every isotropy subgroup $H$ of $B$. When $B^{H}$ is connected for every $H \in \mathcal{H}$, every $G$-fibration over $B$ is a $G$-fibration with the fiber type $\mathcal{F}$. However, a $G$-fibration $p: E \rightarrow B$ does not necessarily have a fiber type.

\section{Tulley's theorem for $G$-fibrations}

The aim of this section is to prove an equivariant version of a theorem due to Tulley (see [16, Theorem 11]). The proof uses the same ideas and methods from [16;15].

Theorem 3.1 Let $p_{1}: E_{1} \rightarrow B$ and $p_{2}: E_{2} \rightarrow B$ be $G$-fiber homotopy equivalent $G$-fibrations. Then there is a $G$-fibration $q$ over $B \times I$ such that $\left.q\right|_{B \times\{0\}}=p_{1}$ and $\left.q\right|_{B \times\{1\}}=p_{2}$.

We call the $G$-fibration $q: Z \rightarrow B \times I$ in Theorem 3.1 a $G$-tube between $p_{1}$ and $p_{2}$. Let $f: E_{1} \rightarrow E_{2}$ be a fiber preserving $G$-map between $G$-fibrations $p_{1}$ and $p_{2}$ over $B$. Recall that the mapping cylinder $M_{f}$ of $f$ is the adjunction space $E_{1} \times I \cup_{f} E_{2}$ where $f(e)=(e, 0)$ for each $e \in E_{1}$. We define the $G$-map $p_{f}: M_{f} \rightarrow B$ over $B$ by $p_{f}(x, s)=p_{1}(x)$ and $p_{f}(y)=p_{2}(y)$ for any $x \in E_{1}, y \in E_{2}$, and $s \in I$.

Lemma 3.2 Let $f: E_{1} \rightarrow E_{2}$ be a fiber preserving $G$-map between $G$-fibrations $p_{1}$ and $p_{2}$ over $B$. Then the induced $G-$ map $p_{f}: M_{f} \rightarrow B$ is a $G$-fibration.

Proof The proof is similar to the proof of [15, Theorem 1]. Let $\lambda_{i}: \Omega_{p_{i}} \rightarrow E_{i}{ }^{I}$ be a $G$-lifting function for $p_{i}, i=1,2$. Since $\Omega_{p_{f}}=\Omega_{p_{1}} \times I \cup \tilde{f} \Omega_{p_{2}}$ where $\tilde{f}(e, \omega)=(f(e), \omega)$, to show that $p_{f}$ is a $G$-fibration it suffices to construct a $G$-map $\lambda: \Omega_{p_{1}} \times I \rightarrow M_{f}^{I}$ such that $\left.\lambda\right|_{\Omega_{p_{1}} \times\{0\}}=\lambda_{2} \circ \tilde{f}, p_{f} \lambda((e, \omega), s)=\omega$, and $\lambda((e, \omega), s)(0)=(e, s)$.

Define $\lambda: \Omega_{p_{1}} \times I \rightarrow M_{f}^{I}$ by

$$
\lambda(e, \omega, s)(t)= \begin{cases}\left(\lambda_{1}(e, \omega)(t), s-t\right) & t \leq s, \\ \lambda_{2}\left(z, \omega^{s}\right)(t-s) & s \leq t,\end{cases}
$$

where $z=f \circ \lambda_{1}(e, \omega)(s)$ and $\omega^{s}$ is given by $\omega^{s}(t)=\omega(s+t)$ when $s+t \leq 1$ and $\omega^{s}(t)=1$, otherwise. Clearly, $\lambda$ is a continuous $G$-map which satisfies the relations $\left.\lambda\right|_{\Omega_{p_{1}}} \times\{0\}=\lambda_{2} \circ \tilde{f}, p_{f} \lambda((e, \omega), s)=\omega$, and $\lambda((e, \omega), s)(0)=(e, s)$. 
In order to prove Theorem 3.1, it suffices to construct $G$-fibrations $q_{1}: Z_{1} \rightarrow B \times I$ and $q_{2}: Z_{2} \rightarrow B \times I$ with $\left.q_{1}\right|_{B \times\{0\}}=p_{1},\left.q_{1}\right|_{B \times\{1\}}=\left.q_{2}\right|_{B \times\{0\}}=p_{f}$ and $\left.q_{2}\right|_{B \times\{1\}}=p_{2}$ where $f$ is the fiber preserving $G$-map between $p_{1}$ and $p_{2}$. That is because once we have such $G$-fibrations, we can obtain a $G$-tube between $p_{1}$ and $p_{2}$ by gluing $q_{1}$ and $q_{2}$ as follows. Let $Z=Z_{1} \cup_{i_{1}} M_{f} \times I \cup_{i_{2}} Z_{2}$ where $i_{1}(x)=(x, 0)$ and $i_{2}(x)=(x, 1)$ for every $x \in M_{f}$. Define the $G$-map $q: Z \rightarrow B \times I$ by

$$
q(z)= \begin{cases}\left(\pi_{1}\left(q_{1}(z)\right), \frac{1}{3} \pi_{2}\left(q_{1}(z)\right)\right) & z \in Z_{1}, \\ \left(\pi_{1}\left(q_{2}(z)\right), \frac{2}{3}+\frac{1}{3} \pi_{2}\left(q_{2}(z)\right)\right) & z \in Z_{2}, \\ \left(p_{f}(x), \frac{1}{3}(1+t)\right) & z=(x, t) \in M_{f} \times I,\end{cases}
$$

where $\pi_{i}$ is the projection map to the $i$-th coordinate. Since we can extend the lifting functions for $q_{1}$ and $q_{2}$ to lifting functions for $\left.q\right|_{B \times[0,6 / 9)}$ and $\left.q\right|_{B \times(4 / 9,1]}$, respectively, $G$-maps $\left.q\right|_{B \times[0,6 / 9)}$ and $q_{B \times(4 / 9,1]}$ are $G$-fibrations. Therefore $q: Z \rightarrow B \times I$ is a $G$-fibration by Theorem 2.3 .

Proposition 3.3 Let $Z_{2}=\left\{(e, s, t) \in E_{1} \times I \times I \mid s+t \leq 1\right\} \cup \tilde{f} E_{2} \times I$ in $M_{f} \times I$, where $\tilde{f}: E_{1} \times\{0\} \times I$ is defined by $\tilde{f}(e, 0, t)=(f(e), t)$. Then $q_{2}=$ $\left.\left(p_{f} \times \mathrm{id}\right)\right|_{Z_{2}}: Z_{2} \rightarrow B \times I$ is a $G$-fibration with $\left.q_{2}\right|_{B \times\{0\}}=p_{f}$ and $\left.q_{2}\right|_{B \times\{1\}}=p_{2}$.

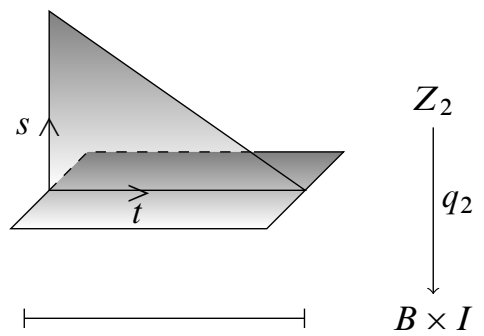

Proof Let $r: M_{f} \times I \rightarrow Z_{2}$ be defined by $\left.r\right|_{E_{2} \times I}=\mathrm{id}_{E_{2} \times I}$ and

$$
\left.r\right|_{E_{1} \times I \times I}(x, s, t)= \begin{cases}(x, s, t) & s+t \leq 1 \\ (x, t, t) & \text { otherwise }\end{cases}
$$

Then $r$ is a fiber preserving $G$-retraction. Let $H: X \times I \rightarrow B$ and $h: X \rightarrow Z_{2}$ be given $G$-maps with $\left.H\right|_{X \times\{0\}}=p_{2} \circ h$. Since $p_{f} \times$ id is a $G$-fibration, there is a 
$G$-map $\bar{H}: X \times I \rightarrow M_{f} \times I$ which makes the following diagram commute:

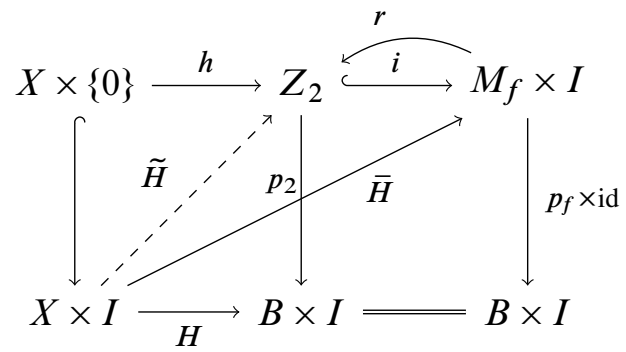

Then the $G$-map $\tilde{H}: X \times I \rightarrow Z_{2}$ defined by $\tilde{H}=r \bar{H}$ satisfies $p_{2} \tilde{H}=H$ and $\left.\widetilde{H}\right|_{X \times\{0\}}=h$.

Definition 3.4 Let $p_{i}: E_{i} \rightarrow B$ be a $G$-fibration for $i=1,2$ with $E_{1} \subseteq E_{2}$ and $\left.p_{2}\right|_{E_{1}}=p_{1}$. Then $p_{1}$ is said to be a $G$-deformation retract of $p_{2}$ if $E_{1}$ is a $G$-deformation retract of $E_{2}$ via fiber preserving $G$-retraction, that is, if there is a $G$-map $H: E_{2} \times I \rightarrow E_{2}$ such that $H_{0}=\operatorname{id}_{E_{2}}, H(e, 1) \in E_{1}$ and $p_{2} H(e, t)=p_{2}(e)$ for every $e \in E_{2}$. If $H$ also satisfies the relation $H(e, t)=e$ for every $e \in E_{1}$, we say $p_{1}$ is a strong $G$-deformation retract of $p_{2}$.

To show that there is a $G$-tube $q_{1}: Z_{1} \rightarrow B \times I$ between $p_{1}$ and $p_{f}$, we need the following proposition. The nonequivariant version of this proposition is proved by Tulley [16] and used in a recent paper by Steimle [13].

Proposition 3.5 If $p_{1}$ is a strong $G$-deformation retract of $p_{2}$ then the $G$-map $q=\left.\left(p_{2} \times \mathrm{id}\right)\right|_{Z}: Z \rightarrow B \times I$ where $Z=\left\{(e, t) \in E_{2} \times I \mid e \in E_{1}\right.$ when $\left.t>0\right\}$ is a $G$-fibration.

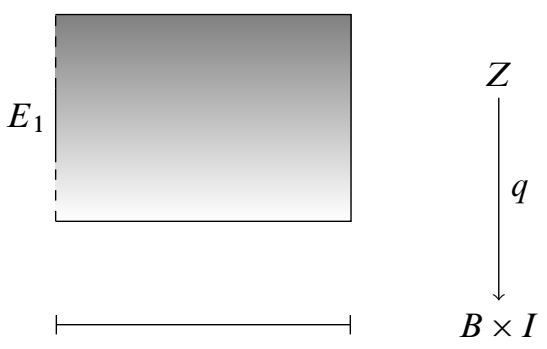

Proof We are using the same approach that is used in the proof of Theorem 3.1 in Langston [7]. Let $H: E_{2} \times I \rightarrow E_{2}$ be a strong $G$-deformation retraction of the 
$G$-fibration $p_{2}$ onto $p_{1}$. Let $\lambda: \Omega_{p_{2}} \rightarrow E_{2}^{I}$ be a $G$-lifting function for $p_{2}$. Define a $G-$ map $\lambda^{\prime}: \Omega_{q} \rightarrow Z^{I}$ by $\pi_{2}\left(\lambda^{\prime}\left(\left(e, \omega_{2}(0)\right),\left(\omega_{1}, \omega_{2}\right)\right)(t)\right)=\omega_{2}(t)$ and $\pi_{1}\left(\lambda^{\prime}\left(\left(e, \omega_{2}(0)\right),\left(\omega_{1}, \omega_{2}\right)\right)(t)\right)= \begin{cases}H\left(\lambda\left(e, \omega_{1}\right)(t), t / \omega_{2}(t)\right) & \omega_{2}(t)>0, \omega_{2}(t) \geq t \\ e & t=\omega_{2}(t)=0, \\ H\left(\lambda\left(e, \omega_{1}\right)(t), 1\right) & t>0, t \geq \omega_{2}(t) .\end{cases}$ Clearly, $q \lambda^{\prime}\left(\left(e, \omega_{2}(0)\right),\left(\omega_{1}, \omega_{2}\right)\right)=\left(\omega_{1}, \omega_{2}\right)$ and $\lambda^{\prime}\left(\left(e, \omega_{2}(0)\right),\left(\omega_{1}, \omega_{2}\right)\right)(0)=e$. Therefore we only need to check the continuity of $\pi_{1} \lambda^{\prime}$ at $t=0$. For this it suffices to show that the adjoint map $\widetilde{\pi_{1} \lambda^{\prime}}: \Omega_{q} \times I \rightarrow E_{2}$ is continuous at $t=0$.

Let $\left(e_{\alpha}, \omega_{1, \alpha}, \omega_{2, \alpha}, t_{\alpha}\right)$ be a net converging to $\left(e, \omega_{1}, \omega_{2}, 0\right)$. Let $U$ be an open neighborhood of $e \in E_{1}$. Since $H: E_{2} \times I \rightarrow E_{2}$ is continuous, $V=H^{-1}(U)$ is open. Since $(e, t) \in V$ for every $t \in I$, there are open neighborhoods $A_{t} \ni e$ and $V_{t} \ni t$ such that $A_{t} \times V_{t} \subseteq V$, for all $t \in I$. Since $I$ is compact, there exist $t_{1}, \ldots, t_{n}$ such that $I=\bigcup_{i=1}^{n} V_{t_{i}}$. Then $A=\bigcap_{i=0}^{n} A_{t_{i}}$ is an open neighborhood of $e$ with the property that $H(A \times I) \subseteq U$. Since $\lambda$ is continuous, there is $\beta$ such that $\tilde{\lambda}\left(e_{\alpha}, \omega_{1, \alpha}, t_{\alpha}\right) \in A$ for every $\beta>\alpha$. Therefore $\widetilde{\pi_{1} \lambda^{\prime}}\left(e_{\alpha}, \omega_{1, \alpha}, \omega_{2, \alpha}, t_{\alpha}\right) \in U$ for every $\alpha>\beta$ as desired.

It is proved in [11, Corollary 2.4.2] that when $f: E_{1} \rightarrow E_{2}$ is a homotopy equivalence then $E_{1}$ is a strong deformation retract of $M_{f}$. The same proof applies to $G$-fibrations (see [5, Lemma 2.5.2]). Therefore, by Proposition 3.5, there is a $G$-tube $q_{1}$ between $p_{1}$ and $p_{f}$. Note that $p_{2}$ is also a strong $G$-deformation retract of $p_{f}$ and one can also use Proposition 3.5 to construct a $G$-tube between $p_{2}$ and $p_{f}$. This completes the proof of Theorem 3.1. As an immediate corollary, we have:

Corollary 3.6 Let $B_{1}, B_{2}$ and $B$ be topological spaces such that $B \subseteq B_{1} \cap B_{2}$. If $p_{1}: E_{1} \rightarrow B_{1}$ and $p_{2}: E_{2} \rightarrow B_{2}$ are $G$-fibrations with $\left.\left.p_{1}\right|_{B} \simeq_{G} p_{2}\right|_{B}$ then there is a $G$-fibration over $B_{1} \cup_{i_{1}}(B \times I) \cup_{i_{2}} B_{2}$ extending $p_{1}$ and $p_{2}$ where $i_{j}: B \times\{j\} \rightarrow B_{j}$ are inclusions.

Proof By Theorem 3.1, there is a $G$-tube $q: Z \rightarrow B \times I$ between $\left.p_{1}\right|_{B}$ and $\left.p_{2}\right|_{B}$. Without loss of generality, we can consider $q$ over $B \times\left[\frac{1}{3}, \frac{2}{3}\right]$ with $\left.q\right|_{B \times\{1 / 3\}}=p_{1}$ and $\left.q\right|_{B \times\{2 / 3\}}=p_{2}$. Let

$$
\tilde{Z}=E_{1} \cup_{k_{1}}\left(p_{1}^{-1}(B) \times\left[0, \frac{1}{3}\right]\right) \cup_{m_{1}} Z \cup_{m_{2}}\left(p_{2}^{-1}(B) \times\left[\frac{2}{3}, 1\right]\right) \cup_{k_{2}} E_{2},
$$

where $k_{1}: p_{1}^{-1}(B) \times\{0\} \rightarrow E_{1}, k_{2}: p_{1}^{-1}(B) \times\{1\} \rightarrow E_{2}$ and $m_{1}: p_{1}^{-1}(B) \times\left\{\frac{1}{3}\right\} \rightarrow Z$, $m_{2}: p_{2}^{-1}(B) \times\left\{\frac{2}{3}\right\} \rightarrow Z$ are the inclusions. Define a $G-$ map

$$
\tilde{q}: \tilde{Z} \rightarrow B_{1} \cup_{i_{1}}(B \times I) \cup_{i_{2}} B_{2}
$$


by $\left.\tilde{q}\right|_{E_{i}}=p_{i},\left.\tilde{q}\right|_{Z \times[1 / 3,2 / 3]}(z, t)=q(z)$ and for $j=1,2$ by $\tilde{q}(e, t)=p_{j}(e)$ when $t \in[2(j-1) / 3,(2 j-1) / 3])$.

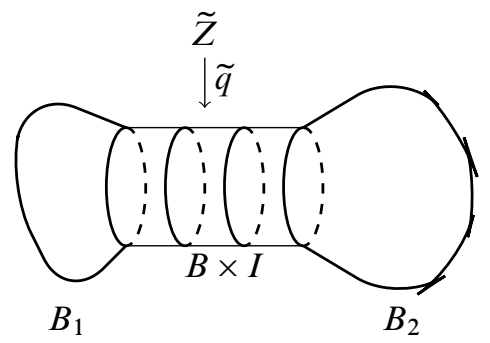

Clearly the restriction of $\widetilde{q}$ to the following subsets are $G$-fibrations

$$
\left\{B_{1} \cup_{i_{1}} B \times\left[0, \frac{2}{9}\right], B \times\left[\frac{1}{9}, \frac{5}{9}\right], B \times\left[\frac{4}{9}, \frac{8}{9}\right], B \times\left[\frac{7}{9}, 1\right] \cup_{i_{2}} E_{2}\right\} .
$$

Therefore, by Theorem 2.3, $\widetilde{q}$ is a $G$-fibration.

Theorem 3.7 Let $p_{i}: E_{i} \rightarrow B, i=1,2$, be $G$-fiber homotopy equivalent $G-$ fibrations such that $E_{1}$ and $E_{2}$ have the $G$-homotopy type of a $G-C W$-complex. Then there is a $G$-tube $q$ between $p_{1}$ and $p_{2}$ whose total space is $G$-homotopy equivalent to a $G-C W-$ complex.

Proof Let $f: E_{1} \rightarrow E_{2}$ be a $G$-fiber homotopy equivalence. Recall that the total space of the $G$-tube $q$ we constructed is $Z=Z_{1} \cup_{i_{1}} M_{f} \times\left[\frac{1}{3}, \frac{2}{3}\right] \cup Z_{2}$ where

$$
\begin{aligned}
& Z_{1}=\left\{(e, t) \in M_{f} \times\left[0, \frac{1}{3}\right] \mid e \in M_{f} \text { if } 0<t \leq \frac{1}{3}, e \in E_{1} \text { if } t=0\right\}, \\
& Z_{2}=\left\{(x, s, t) \in E_{1} \times I \times\left[\frac{2}{3}, 1\right] \mid s+3 t \leq 3\right\} \cup_{f} E_{2} \times\left[\frac{2}{3}, 1\right] .
\end{aligned}
$$

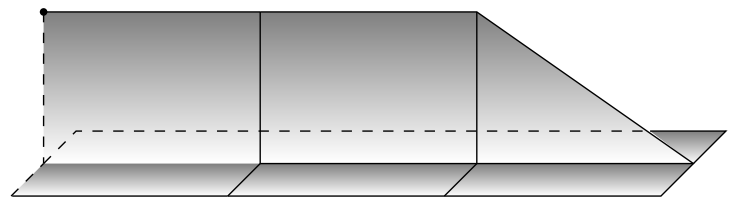

Clearly, $Z$ is a strong $G$-deformation retract of $M_{f}$. On the other hand, $M_{f}$ is $G$-homotopic to $E_{2}$ and hence it has a $G$-homotopy type of a $G-\mathrm{CW}$-complex.

Corollary 3.8 A $G$-fibration $p: E \rightarrow \mathbb{S}^{n-1}$ over $(n-1)$-sphere with trivial $G$-action on the base extends to a $G$-fibration over a disk if and only if it is $G$-fiber homotopy equivalent to a trivial $G$-fibration.

Proof Since $\mathbb{D}^{n}$ is contractible, the "only if" part holds. For the "if" part, let $p$ be $G$-fiber homotopy equivalent to a trivial fibration. Consider $\mathbb{D}^{n}$ as the cone of $\mathbb{S}^{n-1}$, 
that is, $\mathbb{D}^{n}=\mathbb{S}^{n-1} \times[0,2] / \sim$ where $(y, 2) \sim *$. Let $B_{1}=S^{n-1} \times[1,2] / \sim$, and $B_{2}=B=\mathbb{S}^{n-1} \times\{1\}$. Then $\left.\varepsilon\right|_{B} \simeq p$ where $\varepsilon: B_{1} \times F_{G} \rightarrow B_{1}$ where $F_{G}=p^{-1}(x)$ for some $x \in \mathbb{S}^{n-1}$. By Corollary 3.6, there is a $G$-fibration extending $p$.

Remark In [3], the statement of Corollary 3.8 appears on page 137 but in there the total spaces were attached directly which results in a $G$-quasifibration from which one gets a $G$-fibration by taking the corresponding Hurewicz fibration.

\section{Constructing $G$-fibrations}

In this section, we introduce an obstruction theory for constructing $G$-fibrations over $G-\mathrm{CW}-$ complexes and we prove Theorem 1.1. An adequate cohomology theory to develop such an obstruction theory is Bredon cohomology. Let us first fix some notation for Bredon cohomology. We refer the reader to Bredon [2] and Lück [8] for more detailed information about Bredon cohomology.

Let $G$ be a finite group and $\mathcal{H}$ be a family of subgroups of $G$ which is closed under conjugation and taking subgroups. The orbit category $\operatorname{Or}_{\mathcal{H}}(G)$ relative to the family $\mathcal{H}$ is defined as the category whose objects are left cosets $G / H$ where $H \in \mathcal{H}$ and whose morphisms are $G$-maps from $G / H$ to $G / K$. Recall that any $a \in G$ with $H^{a} \leq K$ induces a $G$-map $\hat{a}: G / H \rightarrow G / K$ defined by $\widehat{a}(H)=a K$ and conversely, any $G$-map from $G / H$ to $G / K$ is of this form.

Let $B$ be a $G-\mathrm{CW}$-complex whose isotropy subgroups are all in $\mathcal{H}$. A coefficient system for the Bredon cohomology is a contravariant functor $M: \operatorname{Or}_{\mathcal{H}}(G) \rightarrow \mathcal{A} b$, where $\mathcal{A} b$ is the category of abelian groups. A morphism $T: M \rightarrow N$ between two coefficient systems is a natural transformation of functors. Note that a coefficient system is a $\mathbb{Z} \operatorname{Or}_{\mathcal{H}}(G)$-module with the usual definition of modules over a small category. Since the $\mathbb{Z} \operatorname{Or}_{\mathcal{H}}(G)$-module category is an abelian category, the usual notions for doing homological algebra exist.

Given a local coefficient system $M: \operatorname{Or}_{\mathcal{H}}(G) \rightarrow \mathcal{A} b$, one defines the cochain complex $C_{\mathcal{H}}^{*}(B ; M)$ of $B$ with coefficients in $M$ as follows: Let $C_{\mathcal{H}}^{n}(B ; M)$ be the submodule of $\bigoplus_{H \in \mathcal{H}} \operatorname{Hom}_{\mathbb{Z}}\left(C_{n}\left(B^{H} ; \mathbb{Z}\right), M(G / H)\right)$ formed by elements $(f(H))_{H \in \mathcal{H}}$ which makes the following diagram commute:

$$
\begin{array}{cc}
C_{n}\left(B^{K} ; \mathbb{Z}\right) \stackrel{f(K)}{\longrightarrow} M(G / K) \\
\bar{a}_{n} \downarrow & M(\hat{a}) \downarrow \\
C_{n}\left(B^{H} ; \mathbb{Z}\right) \stackrel{f(H)}{\longrightarrow} M(G / H)
\end{array}
$$


for any $H, K \in \mathcal{H}$ with $H^{a} \leq K$. Here $\bar{a}: B^{K} \rightarrow B^{H}$ is given by $\bar{a}(x)=a x$ for any $x \in B^{K}$ and $\bar{a}_{*}$ denotes the induced map between the chain complexes. The coboundary $\operatorname{map} \delta: C_{\mathcal{H}}^{n}(B ; M) \rightarrow C_{\mathcal{H}}^{n+1}(B ; M)$ is defined by $(\delta f)(H)(\tau)=f(H)(\partial \tau)$ for any $H \in \mathcal{H}$ and for any $(n+1)-$ cell $\tau$ of $B^{H}$.

Definition 4.1 The Bredon cohomology $H_{G, \mathcal{H}}^{*}(B ; M)$ of $G-\mathrm{CW}$-complex $B$ with coefficients in $M$ is defined as the cohomology of the cochain complex $C_{\mathcal{H}}^{*}(B ; M)$.

Let $\mathcal{F}=\left\{F_{H}\right\}_{H \in \mathcal{H}}$ be a compatible family. Since $\mathcal{F}$ is compatible, we can consider the universal $K$-fibration $u_{K}: E_{K} \rightarrow B \operatorname{Aut}_{K}^{I}\left(F_{K}\right)$ with trivial $K$-action on the base as an $H$-fibration with the fiber $F_{H}$ via conjugation action whenever $H^{a} \leq K$. Let $\tilde{a}: B \operatorname{Aut}_{K}^{I}\left(F_{K}\right) \rightarrow B \operatorname{Aut}_{H}^{I}\left(F_{H}\right)$ be the classifying map of this fibration. Define a contravariant functor $\underline{\pi}_{n, \mathcal{F}}: \operatorname{Or}_{\mathcal{H}}(G) \rightarrow \mathcal{A} b$ by letting

$$
\begin{aligned}
\underline{\pi}_{n, \mathcal{F}}(G / H) & =\pi_{n}\left(B \operatorname{Aut}_{H}^{I}\left(F_{H}\right)\right), \\
\underline{\pi}_{n, \mathcal{F}}(\widehat{a}: G / H \rightarrow G / K) & =\pi_{*}(\widetilde{a}): \pi_{n}\left(B \operatorname{Aut}_{K}^{I}\left(F_{K}\right)\right) \rightarrow \pi_{n}\left(B \operatorname{Aut}_{H}^{I}\left(F_{H}\right)\right) .
\end{aligned}
$$

From now on we assume that $B^{H}$ is simply connected for every $H \in \operatorname{Iso}(B)$, where $\operatorname{Iso}(B)$ is the set of isotropy subgroups of $B$. Let $p: E \rightarrow B_{n}$ be a $G$-fibration over the $n$-skeleton of $B$ with the fiber type $\mathcal{F}=\left\{F_{H}\right\}_{H \in \mathcal{H}}$ where $n \geq 2$. For every $H \in \mathcal{H}$, the map $p_{H}: p^{-1}\left(B_{n}^{H}\right) \rightarrow B_{n}^{H}$ is an $H$-fibration. In particular, the $H$-fibration $p_{H}$ is classified by a map $\phi_{p, H}: B_{n}^{H} \rightarrow B \operatorname{Aut}_{H}^{I} F_{H}$ when $H$ is an isotropy subgroup. For an arbitrary $H \in \mathcal{H}$ and $(n+1)-$ cell $\sigma$ of $B_{n}^{H}$ with an attaching map $f_{\sigma}: \mathbb{S}^{n} \rightarrow B_{n}^{G_{\sigma}} \subseteq B_{n}^{H}$, the $G_{\sigma}$-fibration $f_{\sigma}^{*}\left(p_{H}\right)$ is classified by the map $\operatorname{Res}_{H}^{G_{\sigma}} \circ \phi_{p, G_{\sigma}} \circ f_{\sigma}$. Here, $\operatorname{Res}_{H}^{G_{\sigma}}: B$ Aut $_{G_{\sigma}}^{I} F_{G_{\sigma}} \rightarrow B$ Aut $_{H}^{I} F_{H}$ is induced by the relation $H \leq G_{\sigma}$. When $H \in \operatorname{Iso}(\mathrm{B})$, the maps $\operatorname{Res}_{H}^{G_{\sigma}} \circ \phi_{p}, G_{\sigma} \circ f_{\sigma}$ and $\phi_{p, H} \circ f_{\sigma}$ are homotopic since they both classify the $H$-fibration $f_{\sigma}^{*}\left(p_{H}\right)$. Moreover, when $H, K \in \mathcal{H}$ with $H^{a} \leq K$, we have $\widetilde{a} \circ \operatorname{Res}_{K}^{G_{\sigma}} \simeq \operatorname{Res}_{H}^{G_{a \sigma}} \circ \widetilde{a}$ for every $(n+1)$-cell $\sigma$ of $B^{K}$.

We define

$$
\alpha_{p} \in \prod_{H \in \mathcal{H}} \operatorname{Hom}_{\mathbb{Z}}\left(C_{n+1}\left(B^{H}\right), \pi_{n}\left(B \operatorname{Aut}_{H}^{I} F_{H}\right)\right)
$$

by $\alpha_{p}(H)(\sigma)=\left(\operatorname{Res}_{H}^{G_{\sigma}}\right)_{*}\left[\phi_{p}, G_{\sigma} \circ f_{\sigma}\right]$ for every $H \in \mathcal{H}$ and for every $(n+1)-$ cell $\sigma$ of $B^{H}$ with an attaching map $f_{\sigma}: \mathbb{S}^{n} \rightarrow B_{n}^{G_{\sigma}}$. For $\alpha_{p}$ to be a cochain, the following 
diagram must commute up to homotopy

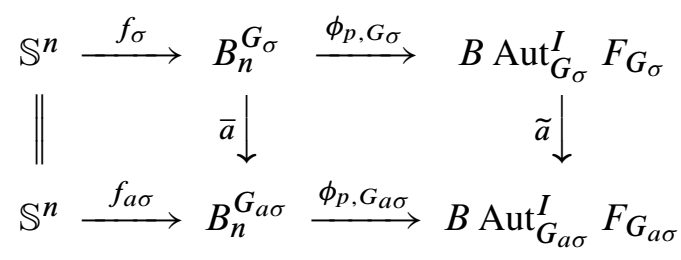

for every $a \in G$. The first square commutes because $B$ has a $G-\mathrm{CW}-$ complex structure. Since $\tilde{a}$ is the classifying map of the $G_{a \sigma}$-fibration $u_{G_{\sigma}}$ and $\phi_{p, G_{\sigma}}$ is the classifying map of $p_{G_{\sigma}}$, the pullback of the universal $G_{a \sigma}$-fibration $u_{G_{a \sigma}}$ by the composition $\tilde{a} \circ \phi_{p, G_{\sigma}}$ is $G_{a \sigma}$-fiber homotopy equivalent to the fibration $p_{G_{\sigma}}$ considered as an $G_{a \sigma}$-fibration. On the other hand, the pullback of the $G_{a \sigma}$-fibration $p_{G_{a \sigma}}$ by $\bar{a}$ is $G_{a \sigma}$-fiber homotopy equivalent to the fibration $p_{G_{\sigma}}$, so $\left(\phi_{p, G_{a \sigma}} \circ \bar{a}\right)^{*} u_{G_{a \sigma}} \simeq G_{a \sigma} p_{G_{\sigma}}$. Therefore the $G_{a \sigma}$-fibrations $\left(\tilde{a} \circ \phi_{p, G_{\sigma}}\right)^{*} u_{G_{a \sigma}}$ and $\left(\phi_{p, G_{a \sigma}} \circ \bar{a}\right)^{*} u_{G_{a \sigma}}$ are $G_{a \sigma}$-fiber homotopy equivalent. By Theorem 2.4, the maps $\tilde{a} \circ \phi_{p, G_{\sigma}}$ and $\phi_{p, G_{a \sigma}} \circ \bar{a}$ are homotopic and hence $\alpha_{p}$ is a cochain in $C_{\mathcal{H}}^{n+1}\left(B, \underline{\pi}_{n, \mathcal{F}}\right)$.

Proposition $4.2 \alpha_{p}$ is a cocycle.

Proof For an $H \in \operatorname{Iso}(B), \alpha_{p}(H) \in C^{n+1}\left(B^{H}, \pi_{n}\left(B \operatorname{Aut}_{H}^{I} F_{H}\right)\right)$ is the obstruction cochain for extending the map $\phi_{p, H}: B_{n}^{H} \rightarrow B$ Aut $_{H}^{I} F_{H}$ to the $(n+1)$-skeleton of $B^{H}$. Therefore, by classical obstruction theory, we have

$$
\left(\delta \alpha_{p}\right)(H)(\tau)=\delta\left(\alpha_{p}(H)\right)(\tau)=0
$$

for any $(n+2)$-cell $\tau$ of $B^{H}$. On the other hand, for arbitrary $H \in \mathcal{H}$, we have $\left(\delta \alpha_{p}\right)(H)(\tau)=\left(\operatorname{Res}_{H}^{G_{\tau}}\right)_{*}\left(\delta \alpha_{p}\left(G_{\tau}\right)(\tau)\right)=0$. So, $\delta \alpha_{p}=0$.

We call $\alpha_{p}$ the obstruction cocycle. From now on we assume that the total spaces of $G$-fibrations that we consider have the homotopy type of a $G-\mathrm{CW}-$ complex unless otherwise stated.

Proposition 4.3 A $G$-fibration $p: E_{n} \rightarrow B_{n}$ with the fiber type $\mathcal{F}=\left\{F_{H}\right\}_{H \in \mathcal{H}}$, where $F_{H}$ is a finite $H-C W$-complex, extends to a $G$-fibration over the $(n+1)$ skeleton $B_{n+1}$ if and only if $\alpha_{p}=0$. Moreover if $E_{n}$ has the $G$-homotopy type of a finite $G-C W$-complex then the total space of the fibration that we obtain has the $G$-homotopy type of a finite $G-C W$-complex.

Proof If the obstruction cocycle is zero, then $\left[\phi_{G_{\sigma}} \circ f_{\sigma}\right]=0$ for any $(n+1)$-cell $\sigma$ of $B$. By Theorem 2.4, the restriction $\left.p\right|_{\partial \sigma}$ is $G_{\sigma}$-fiber homotopy equivalent to 
the trivial $G_{\sigma}$-fibration $\varepsilon: \partial \sigma \times F_{G_{\sigma}} \rightarrow \partial \sigma$. Let $\beta_{\sigma}: \partial \sigma \times F_{G_{\sigma}} \rightarrow p^{-1}(\partial \sigma)$ be the $G_{\sigma}$-fiber homotopy equivalence between them. By Corollary 3.8, $\left.p\right|_{\partial \sigma}$ extends to a $G_{\sigma}$-fibration $\tilde{p}_{\sigma}: Z \rightarrow \sigma$ over $\sigma$. Let us define

$$
E^{\prime}=\left(\coprod_{\sigma \in I_{n+1}} G \times_{G_{\sigma}}\left(Z \cup_{i_{1}}\left(p^{-1}(\partial \sigma) \times I\right)\right)\right) \cup_{i_{2}} E,
$$

where $I_{n+1}$ is the set of representatives of $G$-orbits of $(n+1)$-cells and $i_{j}$ 's are the corresponding inclusions for $j=1,2$. Let $q: E^{\prime} \rightarrow B_{n+1}$ be defined by relations

$$
\left.q\right|_{Z}=\tilde{p}_{\sigma},\left.q\right|_{p^{-1}(\partial \sigma) \times I}=\left.p\right|_{\partial \sigma} \times \text { id } \text { and }\left.q\right|_{E}=p .
$$

By Theorem 2.3, the $G$-map $q$ is a $G$-fibration.

Since all the fibers and the base space have the $G$-homotopy type of a $G-\mathrm{CW}$-complex, $E^{\prime}$ is $G$-homotopy equivalent to a $G-\mathrm{CW}$-complex. More precisely, for each orbit representative $\sigma \in I_{n+1}$, we can deform $G \times_{G_{\sigma}}\left(Z \cup_{i_{1}}\left(p^{-1}(\partial \sigma) \times I\right)\right) \cup_{i_{2}} E$ to $G \times{ }_{G_{\sigma}}\left(\sigma \times F_{G_{\sigma}}\right) \cup_{\beta_{\sigma}} E$ relative to $E$ via a $G$-map as shown in Figure 1 .

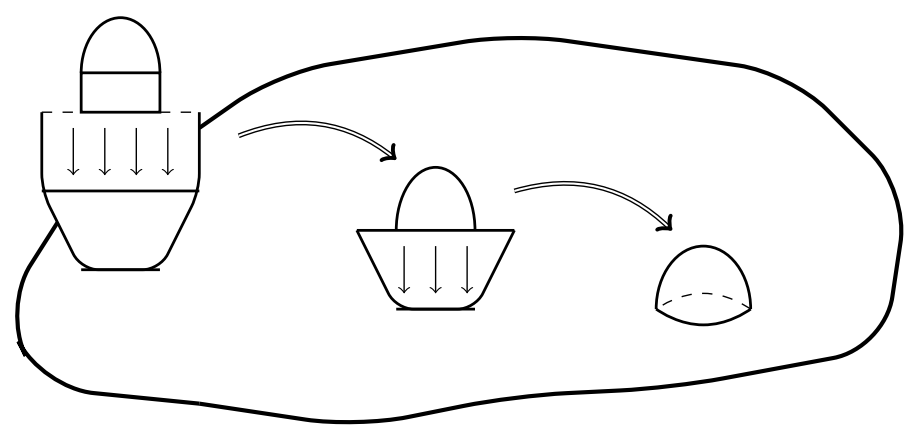

Figure 1

Proposition 4.3 proves the first part of Theorem 1.1. The second part of the theorem says that if $\alpha_{p}$ is cohomologous to zero, that is, $\alpha_{p}=\delta d$ for some cochain $d \in C_{\mathcal{H}}^{n}\left(B, \underline{\pi}_{n, \mathcal{F}}\right)$ then the $G$-fibration $\left.p\right|_{B_{n-1}}: p^{-1}\left(B_{n-1}\right) \rightarrow B_{n-1}$ extends to a $G$-fibration over $B_{n+1}$. In order to prove this, we redefine $p$ over the $n$-skeleton relative to the $(n-1)$-skeleton in such a way that the obstruction cocycle of this new $G$-fibration vanishes.

Let $p_{1}$ and $p_{2}$ be $G$-fibrations over $B_{n}$ whose restrictions to $B_{n-1}$ are $G$-fiber homotopy equivalent. Then by Corollary 3.6, there is a $G$-fibration $q: Z \rightarrow(B \times I)_{n}$ with $\left.q\right|_{B_{n} \times\{0\}}=p_{1}$ and $\left.q\right|_{B_{n} \times\{1\}}=p_{2}$. Let $\Psi_{q, H}:\left(B^{H} \times I\right)_{n} \rightarrow B$ Aut $_{H}^{I} F_{H}$ be the classifying map of the fibration $q_{H}$ for $H \in \operatorname{Iso}(B)$. Note that the composition $\Psi_{q, H} \circ i_{j}$ gives a classifying map for the $H$-fibration $p_{j}^{H}$ where $i_{j}$ 's are the corresponding inclusions. As before, the map $\operatorname{Res}_{H}^{G_{a e}} \circ \Psi_{q, G_{a e}} \circ f_{a e}$ is homotopic to $\tilde{a} \circ \operatorname{Res}_{K}^{G_{e}} \circ \Psi_{q, G_{e}} \circ f_{e}$ for 
every $(n+1)$-cell $e$ of $B^{K} \times I$ with the attaching map $f_{e}: \mathbb{S}^{n-1} \rightarrow B_{n}^{G_{\sigma}}$ and for every $H^{a} \leq K$. Therefore, the map $d_{p_{1}, q, p_{2}} \in \prod_{H \in \mathcal{H}} \operatorname{Hom}_{\mathbb{Z}}\left(C_{n}\left(B^{H}\right), \pi_{n}\left(B \operatorname{Aut}_{H}^{I} F_{H}\right)\right)$ defined for an $n$-cell $\tau$ of $B^{H}$ by

$$
d_{p_{1}, q, p_{2}}(H)(\tau)=(-1)^{n+1}\left(\operatorname{Res}_{H}^{G_{\tau}}\right)_{*}\left[\Psi_{q, G_{\tau}} \circ f_{\tau \times I}\right],
$$

is a cochain in $C_{\mathcal{H}}^{n}\left(B ; \underline{\pi}_{n, \mathcal{F}}\right)$. We call $d_{p_{1}, q, p_{2}}$ the difference cochain. As in the standard theory, we have the following.

Proposition $4.4 \delta d_{p_{1}, q, p_{2}}=\alpha_{p_{1}}-\alpha_{p_{2}}$.

Proof It suffices to show that $d_{p_{1}, q, p_{2}}(H)=\alpha_{p_{1}}(H)-\alpha_{p_{2}}(H)$ for every $H \in \operatorname{Iso}(B)$. Let $o_{\Psi} \in C^{n+1}\left(B^{H} \times I, \pi_{n}\left(B\right.\right.$ Aut $\left.\left._{H}^{I} F_{H}\right)\right)$ be the obstruction cocycle to the extension of $\Psi_{q, H}$ to $\left(B^{H} \times I\right)_{n+1}$. Then $\delta o_{\Psi}=0$. On the other hand, $o_{\Psi}(H)(\theta)=\left[\Psi_{q, H} \circ f_{\theta}\right]$ for every $(n+1)-$ cell $\theta$ of $B^{H} \times I$. Therefore, as in the proof of the corresponding result in the standard theory, we have

$$
\begin{aligned}
0 & =\left(\delta o_{\Psi}\right)(H)(\sigma \times I) \\
& =o_{\Psi}(H)(\partial \sigma \times I)+(-1)^{n+1}\left(o_{\Psi}(H)(\sigma \times\{1\})-o_{\Psi}(H)(\sigma \times\{0\})\right) \\
& =\left[\Psi_{q, H} \circ f_{\partial \sigma \times I}\right]+(-1)^{n+1}\left(\left[\Psi_{q, H} \circ f_{\sigma \times\{1\}}\right]-\left[\Psi_{q, H} \circ f_{\sigma \times\{0\}}\right]\right) \\
& =(-1)^{n+1}\left(d_{p_{1}, q, p_{2}}(H)(\partial \sigma)+\alpha_{p_{2}}(H)(\sigma)-\alpha_{p_{1}}(H)(\sigma)\right) \\
& =(-1)^{n+1}\left(\delta d_{p_{1}, q, p_{2}}(H)(\sigma)+\alpha_{p_{2}}(H)(\sigma)-\alpha_{p_{1}}(H)(\sigma)\right)
\end{aligned}
$$

for any $(n+1)$-cell $\sigma$ of $B^{H}$. This implies that for every $H \in G$ and for every $(n+1)-$ cell $\sigma$ of $B^{H}$, we have

$$
\delta d_{p_{1}, q, p_{2}}(H)(\sigma)=\left(\alpha_{p_{1}}-\alpha_{p_{2}}\right)(H)(\sigma)
$$

and hence $\delta d_{p_{1}, q, p_{2}}=\alpha_{p_{1}}-\alpha_{p_{2}}$.

Proposition 4.4 immediately implies the "if" direction of Theorem 1.1(2). To see this, note that if the $G$-fibration $\left.p\right|_{B_{n-1}}$ extends to a $G$-fibration $\tilde{p}$ over $B_{n+1}$ then

$$
\delta d_{p, q,\left.\tilde{p}\right|_{B n}}=\alpha_{p}-\overbrace{\alpha_{\left.\tilde{p}\right|_{B_{n}}}^{0}}^{0}=\alpha_{p},
$$

hence the cohomology class $\left[\alpha_{p}\right]$ vanishes. For the "only if" direction, we need the following observation.

Proposition 4.5 Let $p: E \rightarrow B_{n}$ be a $G$-fibration over $B_{n}$ with the fiber type $\mathcal{F}=$ $\left\{F_{H}\right\}_{H \in \mathcal{H}}$ where $F_{H}$ is a finite $H-C W$-complex for every $H \in \mathcal{H}$. Then for every $d \in C_{\mathcal{H}}^{n}\left(B ; \underline{\pi}_{n, \mathcal{F}}\right)$, there is a $G$-fibration $q: Z \rightarrow(B \times I)_{n}$ such that $d_{p, q, \tilde{p}}=d$ where $\tilde{p}=\left.q\right|_{B_{n} \times\{1\}}$. Moreover if $E$ has the $G$-homotopy type of a finite $G-C W$-complex then the space $q^{-1}\left(B_{n} \times\{1\}\right)$ has the $G$-homotopy type of a finite $G-C W$-complex. 
Proof For an $n$-cell $\tau$ of $B$, the $G_{\tau}$-map $\bar{p}_{\tau}: p^{-1}(\bar{\tau}) \cup p^{-1}(\partial \tau) \times I \rightarrow \bar{\tau} \cup \partial \tau \times I$, where $\left.\bar{p}_{\tau}\right|_{p^{-1}(\bar{\tau})}=\left.p\right|_{p^{-1}(\bar{\tau})}$ and $\left.\bar{p}_{\tau}\right|_{p^{-1}(\partial \tau) \times I}=\left.p\right|_{p^{-1}(\partial \tau)} \times$ id, is a $G_{\tau}$-fibration and it is classified by the map $\phi_{p, G_{\tau}} \circ \pi_{1}$ where $\pi_{1}: \bar{\tau} \cup \partial \tau \times I \rightarrow \bar{\tau}$ is the projection to the first coordinate. Let $E_{\tau}$ be the pullback of $\bar{p}_{\tau}$ by the map

$$
\overline{f_{\tau}}=\left(f_{\tau} \circ \pi_{1}, f_{\tau} \times \mathrm{id}\right): \mathbb{D}^{n} \times\{0\} \cup \mathbb{S}^{n-1} \times I \rightarrow \bar{\tau} \cup \partial \tau \times I
$$

where $f_{\tau}:\left(\mathbb{D}^{n}, \mathbb{S}^{n-1}\right) \rightarrow(\bar{\tau}, \tau)$ is the characteristic map of $\tau$.

Let $X_{1}=\left\{(x, 1) \in \mathbb{D}^{n} \times\{1\}\left|\frac{1}{2} \leq\right| x \mid \leq 1\right\}, X_{2}=\left\{(x, 1) \in \mathbb{D}^{n} \times\{1\}\left|\frac{1}{4} \leq\right| x \mid \leq \frac{1}{2}\right\}$, and $X_{3}=\left\{(x, 1) \in \mathbb{D}^{n} \times\{1\}|0 \leq| x \mid \leq \frac{1}{4}\right\}$. Let $p_{1}: E_{\tau}^{1} \rightarrow X_{1}$ be the induced $G_{\tau}$-fibration $\left(\bar{f}_{\tau} f\right)^{*}\left(\bar{p}_{\tau}\right)$ where $f: X_{1} \rightarrow \mathbb{D}^{n} \times\{0\} \cup \mathbb{S}^{n-1} \times I$ is given by

$$
f(x, 1)= \begin{cases}(2 x-x /|x|, 4|x|-3) & \frac{3}{4} \leq|x| \leq 1, \\ (2 x-x /|x|, 0) & \frac{1}{2} \leq|x| \leq \frac{3}{4} .\end{cases}
$$

Note that $\left.p_{1}\right|_{\mathbb{S}_{1}^{n-1} \times\{1\}}=f_{\tau}^{*}\left(\left.\bar{p}\right|_{\partial \tau}\right)$ and $\left.p_{1}\right|_{\mathbb{S}_{1 / 2}^{n-1} \times\{1\}}$ is the trivial $G_{\tau}$-fibration with the fiber $F=p^{-1}\left(f_{\tau}(0)\right)$, where $\mathbb{S}_{r}^{n-1}$ is the $(n-1)$-sphere of radius $r$.

Since $d\left(G_{\tau}\right)(\tau) \in \pi_{n}\left(B \operatorname{Aut}_{G_{\tau}}^{I} F_{G_{\tau}}\right)$, it is represented by a map

$$
\Psi_{\tau}:\left(\mathbb{D}_{1 / 4}^{n}, \mathbb{S}_{1 / 4}^{n-1}\right) \rightarrow\left(B \operatorname{Aut}_{G_{\tau}}^{I} F_{G_{\tau}}, *\right) .
$$

Let $u_{G_{\tau}}^{\prime}=E \operatorname{Aut}_{G_{\tau}}^{I} F_{G_{\tau}} \times \operatorname{Aut}_{G_{\tau}}^{I} F_{G_{\tau}} F \rightarrow B$ Aut $_{G_{\tau}}^{I} F_{G_{\tau}}$. Then the restriction of the $G_{\tau}$-fibration $\Psi_{\tau}^{*}\left(u_{G_{\tau}}^{\prime}\right): E_{\tau}^{2} \rightarrow X_{3}$ to $\mathbb{S}_{1 / 4}^{n-1} \times\{1\}$ is the same as the trivial $G_{\tau}$-fibration with the fiber $F$. By gluing these fibration with the trivial one over $X_{2}$, we obtain a $G_{\tau}$-fibration $E_{\tau}^{1} \cup F \times X_{2} \cup E_{\tau}^{2} \rightarrow \mathbb{D}^{n} \times\{1\}$ over $\mathbb{D}^{n} \times\{1\}$. Let $\tilde{p}_{\tau}: \widetilde{E}_{\tau} \rightarrow \tau$ be the corresponding $G_{\tau}$-fibration over $\tau$. As in the proof of Proposition 4.3, the $G$-map

$$
\widetilde{E}=\left(\bigsqcup_{\tau \in I_{n}} G \times_{G_{\tau}}\left(\widetilde{E}_{\tau} \cup_{i_{1}} p^{-1}(\partial \tau) \times I\right)\right) \cup_{i_{2}} p^{-1}\left(B_{n-1}\right)
$$

is a $G$-fibration over $B_{n}$. Moreover, when $E$ has the $G$-homotopy type of a $G-\mathrm{CW}-$ complex, so does $\widetilde{E}$.

Let $q: E \cup\left(p^{-1}\left(B_{n-1}\right) \times I\right) \cup \widetilde{E} \rightarrow(B \times I)_{n}$ be the $G$-fibration defined by $\left.q\right|_{E}=p$, $\left.q\right|_{\widetilde{E}}=\tilde{p}$, and $\left.q\right|_{p^{-1}\left(B_{n-1}\right) \times I}=\left.p\right|_{B_{n-1}} \times$ id. Then $d_{p, q, \tilde{p}}\left(G_{\tau}\right)(\tau)$ is represented by the classifying map

$$
\widetilde{\Psi}: \mathbb{D}^{n} \times\{0\} \cup \mathbb{S}^{n-1} \times I \cup X_{1} \cup X_{2} \cup X_{3} \rightarrow B \operatorname{Aut}_{G_{\tau}} F_{G_{\tau}},
$$


where

$$
\begin{aligned}
& \left.\widetilde{\Psi}\right|_{\mathbb{D}^{n} \times\{0\} \cup \mathbb{S}^{n-1} \times I}=\phi_{p, G_{\tau} \pi_{1}} \overline{f_{\tau}},\left.\quad \widetilde{\Psi}\right|_{X_{1}}=\phi_{p, G_{\tau}} \pi_{1} \overline{f_{\tau}} f \\
& \left.\widetilde{\Psi}\right|_{X_{2}}=c_{\phi_{p, G}\left(f_{\tau}(0)\right)},\left.\quad \widetilde{\Psi}\right|_{X_{3}}=\Psi_{\tau} \text {. }
\end{aligned}
$$

Here, $c_{\phi_{p, G_{\tau}}\left(f_{\tau}(0)\right)}$ is the constant map at $\phi_{p, G_{\tau}}\left(f_{\tau}(0)\right)$. Since $\left.\widetilde{\Psi}\right|_{\mathbb{D}^{n} \times\{0\} \cup \mathbb{S}^{n-1} \times I \cup X_{1}}$ is homotopic to the constant map $c_{\phi_{p, G_{\tau}}\left(f_{\tau}(0)\right)}$ relative to $\mathbb{S}_{1 / 2}^{n-1} \times\{1\}$, the classifying map $\widetilde{\Psi}$ also represents $d\left(G_{\tau}\right)(\tau)$. Therefore, we have $d=d_{p, q, \widetilde{p}}$.

Now we can prove the "only if" of the main theorem as follows.

Proof of Theorem 1.1 It only remains to show that if $\alpha_{p}$ is cohomologous to zero then there is a $G$-fibration over $B_{n+1}$ which extends $\left.p\right|_{B_{n-1}}$. Let $\alpha_{p}=\delta d$ for some $d \in \operatorname{Hom}\left(C_{n}(B), \underline{\pi}_{n, \mathcal{F}}\right)$. By Proposition 4.5, there is a $G$-fibration $q$ over $B \times I$ such that $d=d_{p, q, \tilde{p}}$ where $\tilde{p}=\left.q\right|_{B_{n} \times\{1\}}$. Since $\alpha_{p}=\delta d=\alpha_{p}-\alpha_{\tilde{p}}$, we have $\alpha_{\tilde{p}}=0$ and hence $\tilde{p}$ extends to a $G$-fibration over $B_{n+1}$.

Remark In Theorem 1.1, one can replace the assumption that $B^{H}$ is simply connected for every $H \in \mathcal{H}$ with the assumption that the map $\pi_{1}\left(B^{H}\right) \rightarrow\left[F_{H}, F_{H}\right]_{H}$ is trivial for every $H \in \mathcal{H}$. In applications, one often has fibers which are homotopy equivalent to spheres and one can take fiber joins to make this map trivial.

Acknowledgements This work is part of the author's PhD thesis at the Bilkent University. The author is grateful to her thesis advisor Ergün Yalçın for introducing her to this problem, for valuable discussions and for the careful reading of the first draft. The author thanks Özgün Ünlü for his crucial comments on this work. We also thank the referee for helpful comments, in particular, for suggesting a simpler map which shortens the proof of Lemma 3.2.

The author is supported by TÜBİTAK-TBAG/110T712.

\section{References}

[1] A Adem, J H Smith, Periodic complexes and group actions, Ann. of Math. 154 (2001) 407-435 MR1865976

[2] G E Bredon, Equivariant cohomology theories, Lecture Notes in Math. 34, Springer, Berlin (1967) MR0214062

[3] F X Connolly, S Prassidis, Groups which act freely on $\mathbf{R}^{m} \times S^{n-1}$, Topology 28 (1989) 133-148 MR1003578

[4] A Dold, Partitions of unity in the theory of fibrations, Ann. of Math. 78 (1963) 223-255 MR0155330 
[5] A Güçlükan İlhan, Obstructions for constructing $G$-equivariant fibrations, $\mathrm{PhD}$ thesis, Bilkent University (2011) arXiv:1110.3880v2

[6] M Klaus, Constructing free actions of p-groups on products of spheres, Algebr. Geom. Topol. 11 (2011) 3065-3084 MR2869452

[7] S L Langston, Replacement and extension theorems in the theory of Hurewicz fiber spaces, $\mathrm{PhD}$ thesis, University of Wisconsin-Madison (1968)

[8] W Lück, Transformation groups and algebraic $K$-theory, Mathematica Gottingensis, Lecture Notes in Math. 1408, Springer, Berlin (1989) MR1027600

[9] I Madsen, C B Thomas, C T C Wall, The topological spherical space form problemII Existence of free actions, Topology 15 (1976) 375-382 MR0426003

[10] J P May, A concise course in algebraic topology, Chicago Lectures in Math., Univ. of Chicago Press (1999) MR1702278

[11] R A Piccinini, Lectures on homotopy theory, North-Holland Math. Studies 171, NorthHolland, Amsterdam (1992) MR1150242

[12] J Stasheff, A classification theorem for fibre spaces, Topology 2 (1963) 239-246 MR0154286

[13] W Steimle, Higher Whitehead torsion and the geometric assembly map arXiv: 1105.2116v1

[14] R G Swan, Periodic resolutions for finite groups, Ann. of Math. 72 (1960) 267-291 MR0124895

[15] P Tulley McAuley, A note on paired fibrations, Proc. Amer. Math. Soc. 34 (1972) 534-540 MR0423336

[16] P Tulley, A strong homotopy equivalence and extensions for Hurewicz fibrations, Duke Math. J. 36 (1969) 609-619 MR0248834

[17] Ö Ünlü, Constructions of free group actions on products of spheres, PhD thesis, University of Wisconsin-Madison (2004)

[18] S Waner, Equivariant classifying spaces and fibrations, Trans. Amer. Math. Soc. 258 (1980) 385-405 MR558180

[19] S Waner, Equivariant fibrations and transfer, Trans. Amer. Math. Soc. 258 (1980) 369-384 MR558179

Department of Mathematics, Bilkent University

Ankara 06800, Turkey

guclukan@fen.bilkent.edu.tr

http://www.fen.bilkent.edu.tr/ guclukan

Received: 21 October $2011 \quad$ Revised: 13 March 2012 